STRUCTURAL BIOLOGY COMMUNICATIONS

ISSN 2053-230X

\section{Structure of the substrate-binding domain of Plasmodium falciparum heat-shock protein $70-x$}

Julia Schmidt¥ and Ioannis Vakonakis*

Department of Biochemistry, University of Oxford, South Parks Road, Oxford OX1 3QU, United Kingdom. *Correspondence e-mail: ioannis.vakonakis@bioch.ox.ac.uk

Received 29 June 2020

Accepted 1 September 2020

Edited by M. W. Bowler, European Molecular Biology Laboratory, France

₹ Current address: Department of Biochemistry, Julius-Maximilians-Universität Würzburg,

Sanderring 2, 97070 Würzburg, Germany.

Keywords: malaria; chaperones; Plasmodium falciparum; erythrocyte remodelling; PfHsp70-x; PfEMP-1; crystallography; complexes.

PDB reference: Hsp70-x substrate-binding domain complexed with a hydrophobic peptide, 6zhi

Supporting information: this article has supporting information at journals.iucr.org/f

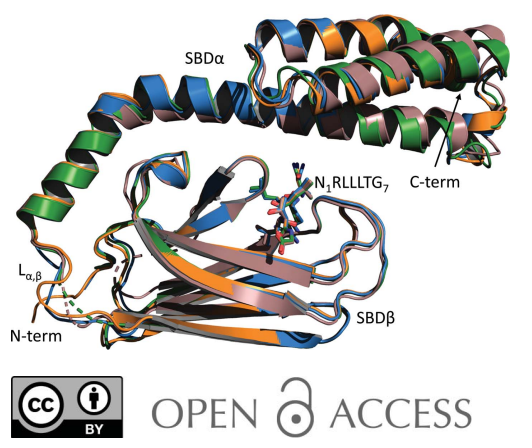

The malaria parasite Plasmodium falciparum extensively modifies erythrocytes that it invades by exporting a large complement of proteins to the host cell. Among these exported components is a single heat-shock $70 \mathrm{kDa}$ class protein, PfHsp70-x, that supports the virulence and growth rate of the parasite during febrile episodes. The ATP-binding domain of PfHsp70-x has previously been resolved and showed the presence of potentially druggable epitopes that differ from those on human Hsp70 chaperones. Here, the crystallographic structure of the substrate-binding domain (SBD) of PfHsp70-x is presented in complex with a hydrophobic peptide. The PfHsp70-x SBD is shown to be highly similar to the counterpart from a human erythrocytic Hsp70 chaperone. The binding of substrate at the interface between $\beta$-sandwich and $\alpha$-helical subdomains of this chaperone segment is also conserved between the malaria parasite and humans. It is hypothesized that the parasite may partly exploit human chaperones for intra-erythrocytic trafficking and maintenance of its exported proteome.

\section{Introduction}

Malaria is an acute febrile illness caused by five different Plasmodium species in humans (World Health Organization, 2019). Of these, $P$. falciparum is the most prevalent and dangerous species, accounting for nearly $95 \%$ of malaria deaths globally. Key to $P$. falciparum virulence is the induction of strong cell adhesion in the erythrocytes that it invades, which is a characteristic of this parasite species among humaninfective Plasmodia (Craig et al., 2012; Smith et al., 2013). The adhesion of parasitized erythrocytes to endothelial cells, such as those in the microvasculature lining of the brain, kidneys or placenta, and to other erythrocytes, forming cell clumps, leads to blood-vessel blockage, tissue damage from oxygen deprivation and inflammation, and potentially to coma and death.

Erythrocyte cytoadherence is the result of extensive hostcell remodelling mediated by parasite proteins exported to the host, collectively referred to as the parasite 'exportome' (Spillman et al., 2015). The exported proteins include the $P$. falciparum erythrocyte membrane protein 1 (PfEMP1) family of adhesion ligands that mediate cell attachment (Hviid \& Jensen, 2015), members of the Plasmodium helical interspersed sub-telomeric (PHIST) protein family that act as interaction hubs (Warncke et al., 2016), and parasite components that remodel the host membrane and cytoskeleton, create nutrient-permeability pathways and contribute to immune-system evasion (de Koning-Ward et al., 2016; Proellocks et al., 2016; Goldberg \& Cowman, 2010). P. falciparum also exports a single heat-shock $70 \mathrm{kDa}$-class protein chaperone, PfHsp70-x (PF3D7_0831700; Külzer et al., 2012), to the host cell. Hsp70-class chaperones play key roles across 
the kingdoms of life in protein quality control, protein translocation, folding and assembly of complexes, and preventing protein aggregation (Boorstein et al., 1994; Meimaridou et al., 2009). PfHsp70-x is found in the parasitophorous vacuole, where it may associate with the PTEX translocation machinery (Zhang et al., 2017), as well as in the host-cell cytoplasm, where it localizes in mobile structures, the 'J-dots', together with stimulatory Hsp40-class co-chaperones and the PfEMP1 adhesion ligand (Külzer et al., 2012). Thus, it is thought that PfHsp70-x assists in PfEMP1 folding and in the assembly of a virulence complex on the erythrocyte membrane that includes PfEMP1. Consistent with this analysis, parasite lines lacking PfHsp70-x exported PfEMP1 less efficiently and were $\sim 60 \%$ less adherent compared with control parasites (Charnaud et al., 2017). Furthermore, in addition to its role in parasite virulence, PfHsp70-x supports cell viability at elevated temperatures, as PfHsp70-x depletion reduced parasite growth by $\sim 40 \%$ during heat shocks comparable to the febrile episodes of malaria patients (Day et al., 2019). These studies have established PfHsp70-x as a key component for parasite survival and virulence.

The function of Hsp70-class chaperones is linked to the binding of exposed hydrophobic segments of aggregationprone proteins, 'holding' these and facilitating their correct folding in a catalytic cycle driven by ATP hydrolysis (Boorstein et al., 1994; Meimaridou et al., 2009). The Hsp70 structure comprises an $\mathrm{N}$-terminal nucleotide-binding domain (NBD) and a C-terminal substrate-binding domain (SBD) subdivided into twisted $\beta$-sandwich $(\operatorname{SBD} \beta)$ and $\alpha$-helical lid $(\operatorname{SBD} \alpha)$ parts; the NBD and SBD temporarily associate as part of the catalytic cycle, which also includes the opening and closing of the $\operatorname{SBD} \beta / \mathrm{SBD} \alpha$ subdomains to trap the unfolded substrate at their interface. We previously resolved the crystallographic structure of the PfHsp70-x NBD as well as that of the cognate parasite co-chaperone PFA0660w (Day et al., 2019), which stimulates PfHsp70-x activity (Daniyan et al., 2016), and used these to create a hybrid model of the chaperone-co-chaperone complex that could be compared with human Hsp70 chaperone structures. Here, we report the PfHsp70-x SBD structure in the domain-closed state complexed with a model substrate peptide, used in previous studies of the human Hsp70 and Escherichia coli DnaK SBDs (Zhang et al., 2014; Zhu et al., 1996), that emulates exposed hydrophobic segments of unstructured or misfolded proteins. We show that the PfHsp70-x SBD is highly similar to the human erythrocytic chaperone Hsp70 in structure and mode of substrate binding.

\section{Materials and methods}

\subsection{Macromolecule production}

A synthetic, codon-optimized DNA fragment (Integrated DNA Technologies) encoding PfHsp70-x residues 424-650 (PfHsp70-x SDB; UniProt K7NTP5) was cloned in the pFLOAT vector (Rogala et al., 2015), which provides an $\mathrm{N}$-terminal $\mathrm{His}_{6}$ tag separated from the target protein by a Human rhinovirus (HRV) 3C protease cleavage site (Table 1).
Table 1

Macromolecule-production information.

\begin{tabular}{ll}
\hline Source organism & P. falciparum \\
DNA source & Synthetic \\
Cloning vector & pFLOAT \\
Expression vector & pFLOAT \\
Expression host & E. coli Rosetta 2(DE3) \\
Complete amino-acid sequence & GPLLDVCPLSLGLETAGGVMTKLIERNTTI \\
of the construct produced & PTKKNQIFTTYADNQPGVLIQVYEGERA \\
& MTKDNNLLGKELEGIPPAPRSVPIEV \\
& TFDIDANGILNVTALDKGTGKQNQITIT \\
& NDKGRLSKDDIDRMVNDAEKYKEEDEQN \\
& KNRIEARNNLENYCYNVKNTLQDENLKT \\
& KIPKDDSEKCMKTVKSVDWLEKNQTAE \\
& TEEYNEKEKDISSVYNPIMTKIYQGASA \\
& QE \\
\hline
\end{tabular}

The plasmid construct was transformed into Escherichia coli Rosetta 2(DE3) competent cells (Novagen). The transformed E. coli cells were used to inoculate lysogeny broth medium supplemented with appropriate antibiotics and the cells were grown at $37^{\circ} \mathrm{C}$ to an $\mathrm{OD}_{600}$ of $\sim 0.6$. Protein expression was induced by the addition of a $0.25 \mathrm{~m} M$ final concentration of isopropyl $\beta$-D-thiogalactopyranoside and was left to proceed at $18^{\circ} \mathrm{C}$ overnight. The cells were harvested by centrifugation and were resuspended in lysis buffer [ $500 \mathrm{mM} \mathrm{NaCl}, 20 \mathrm{mM}$ $\mathrm{Na}_{2} \mathrm{HPO}_{4} \mathrm{pH} 7.2,0.01 \%(v / v)$ Triton X-100]. The cells were lysed by sonication and the lysates were clarified by centrifugation for $60 \mathrm{~min}$ at $25000 \mathrm{~g}$.

The clarified cell supernatants were loaded onto metalaffinity HiTrap Talon columns (GE Healthcare Life Sciences) equilibrated in lysis buffer. The columns were washed using lysis buffer supplemented with $10 \mathrm{~m} M$ imidazole and the proteins were eluted using a gradient of imidazole concentration to $500 \mathrm{~m} M$. Protein-containing fractions were pooled and dialysed against 3C cleavage buffer [ $50 \mathrm{mM} \mathrm{NaCl}, 20 \mathrm{~m} M$ $\mathrm{Na}_{2} \mathrm{HPO}_{4} \mathrm{pH} 7.2,1 \mathrm{~m} M$ dithiothreitol (DTT)] prior to overnight incubation with recombinant HRV $3 \mathrm{C}$ protease engineered with a noncleavable $\mathrm{His}_{6}$ tag. The HRV $3 \mathrm{C}$ protease was removed by a reverse metal-affinity step using a HiTrap Talon column, with PfHsp70-x SBD in the column flowthrough. The PfHsp70-x SBD samples were then loaded onto an ion-exchange HiTrap Q column (GE Healthcare Life Sciences) equilibrated in 3C cleavage buffer and eluted using a $\mathrm{NaCl}$ gradient to a concentration of $500 \mathrm{~m} M$. Protein samples were concentrated by centrifugal ultrafiltration using Amicon Ultra $10 \mathrm{kDa}$ cutoff devices (Merck Millipore) and were loaded onto a Superdex 75 16/600 HiLoad column (GE Healthcare Life Sciences) equilibrated in crystallization buffer [150 $\mathrm{m} M \quad \mathrm{NaCl}, \quad 20 \mathrm{mM}$ 4-(2-hydroxyethyl)-1-piperazineethanesulfonic acid (HEPES) $\mathrm{pH} 7.2,1 \mathrm{~m} M$ DTT]. The protein-containing samples were pooled and concentrated as described above.

The protein quality was analysed by SDS-PAGE. The protein concentration was estimated by UV absorption at $280 \mathrm{~nm}$ using a Nanodrop spectrophotometer (Thermo Scientific) and a modified extinction coefficient calculated from the expected amino-acid sequence (Pace et al., 1995). All chemicals were purchased from Sigma-Aldrich unless otherwise noted. 
Table 2

Crystallization.

\begin{tabular}{|c|c|}
\hline Method & Sitting-drop vapour diffusion \\
\hline Temperature (K) & 293 \\
\hline Protein concentration & $\begin{array}{l}10 \mathrm{mg} \mathrm{ml}^{-1} \text { protein }+5 \mathrm{~m} M \text { NRLLLTG } \\
\text { peptide }\end{array}$ \\
\hline $\begin{array}{l}\text { Buffer composition of protein } \\
\text { solution }\end{array}$ & $\begin{array}{l}150 \mathrm{~m} M \mathrm{NaCl}, 20 \mathrm{~m} M \text { HEPES pH 7.2, } \\
1 \mathrm{~m} M \text { DTT }\end{array}$ \\
\hline $\begin{array}{l}\text { Composition of reservoir } \\
\text { solution }\end{array}$ & $\begin{array}{l}0.1 M \text { bicine/Trizma base pH } 8.5,10 \%(w / v) \\
\text { PEG } 20000,20 \%(w / v) \text { PEG MME } 550, \\
0.03 M \text { of each of diethylene glycol to } \\
\text { pentaethylene glycol }\end{array}$ \\
\hline Volume and ratio of drop & $200 \mathrm{nl}, 1: 1$ protein:mother liquor \\
\hline Volume of reservoir $(\mu \mathrm{l})$ & 40 \\
\hline
\end{tabular}

Table 3

Data collection and processing.

Values in parentheses are for the outer shell.

\begin{tabular}{ll}
\hline Diffraction source & Beamline I03, Diamond Light Source \\
Wavelength $(\AA)$ & 0.9793 \\
Temperature (K) & 100 \\
Detector & PILATUS3 6M, Dectris \\
Rotation range per image $\left({ }^{\circ}\right)$ & 0.1 \\
Total rotation range $\left({ }^{\circ}\right)$ & 360 \\
Exposure time per image (s) & 0.01 \\
Space group & $P 2_{1}$ \\
$a, b, c(\AA)$ & $71.07,93.85,85.30$ \\
$\alpha, \beta, \gamma\left({ }^{\circ}\right)$ & $90,99.47,90$ \\
Resolution range $(\AA)$ & $93.85-3.25(3.31-3.25)$ \\
Total No. of reflections & $120016(5297)$ \\
No. of unique reflections & $17457(796)$ \\
Completeness $(\%)$ & $99.8(94.1)$ \\
Multiplicity & $6.9(6.7)$ \\
$\langle I / \sigma(I)\rangle$ & $8.7(1.5)$ \\
$R_{\text {p.i.m. }}$ & $0.048(0.777)$ \\
$C C_{1 / 2}$ & $0.997(0.568)$ \\
Overall $B$ factor from Wilson plot & 94.35
\end{tabular}

$\left(\AA^{2}\right)$

\subsection{Crystallization}

A synthetically produced heptapeptide with the sequence $\mathrm{N}_{1}$ RLLLTG $_{7}$ (GL Biochem, Shanghai, People's Republic of China) was dissolved directly in crystallization buffer. Crystallization samples formulated in the same buffer were composed of PfHsp70-x SBD and this peptide at final concentrations of $10 \mathrm{mg} \mathrm{ml}^{-1}$ and $5 \mathrm{mM}$, respectively. Samples were incubated for $15 \mathrm{~min}$ at $42^{\circ} \mathrm{C}$, gradually cooled to room temperature and centrifuged at $15000 \mathrm{~g}$ for $5 \mathrm{~min}$ prior to crystallization-drop setup using the sitting-drop vapourdiffusion method. Crystallization drops of $200 \mathrm{nl}$ final volume were set up using a Mosquito robot (TTP Labtech) and a 1:1 ratio of protein-containing solution to mother liquor (Table 2). Diffracting crystals developed after incubation at $20^{\circ} \mathrm{C}$ for three days with a mother liquor consisting of $0.1 \mathrm{M}$ bicine/ 2-amino-2-(hydroxymethyl)propane-1,3-diol (Trizma) base $\mathrm{pH}$ $8.5,10 \%(w / v)$ polyethylene glycol (PEG) $20000,20 \%(w / v)$ PEG MME 550 and $0.03 \mathrm{M}$ each of an ethylene glycol mixture (diethylene glycol to pentaethylene glycol).

\subsection{Data collection and processing}

Diffraction data were collected on beamline I03 at Diamond Light Source, Harwell, UK to a resolution of $3.25 \AA$.
Table 4

Structure refinement.

Values in parentheses are for the outer shell.

\begin{tabular}{ll}
\hline Resolution range $(\AA)$ & $84.13-3.25(3.28-3.25)$ \\
Completeness $(\%)$ & 99.7 \\
$\sigma$ Cutoff & $F>0.000 \sigma(F)$ \\
No. of reflections, working set & $17437(399)$ \\
No. of reflections, test set & 843 \\
Final $R_{\text {cryst }}$ & $0.297(0.289)$ \\
Final $R_{\text {free }}$ & $0.321(0.299)$ \\
No. of non-H atoms & \\
$\quad$ Protein & 7012 \\
$\quad$ Ligand & 0 \\
$\quad$ Water & 0 \\
R.m.s. deviations & \\
$\quad$ Bonds $(\AA)$ & 0.009 \\
Angles $\left({ }^{\circ}\right)$ & 1.12 \\
Average $B$ factors $\left(\AA^{2}\right)$ & 152.5 \\
Protein geometry & \\
$\quad$ Ramachandran favoured $(\%)$ & 99.54 \\
$\quad$ Ramachandran disallowed $(\%)$ & 0.00 \\
Favoured rotamers $(\%)$ & 99.5 \\
Poor rotamers $(\%)$ & 0.00 \\
MolProbity clashscore, all atoms & 15.1 \\
MolProbity clashscore, percentile & 97 th \\
MolProbity score & 1.68 \\
MolProbity score, percentile & 100 th \\
\hline
\end{tabular}

Diffraction data were processed with xia2 in DIALS (Winter, 2010; Winter et al., 2018). The space group was determined to be $P 12{ }_{1} 1$, with four copies per asymmetric unit (data statistics are given in Table 3 ).

\subsection{Structure solution, refinement and analysis}

The structure was solved by molecular replacement using Phaser (McCoy et al., 2007) with a single copy of the human Hsp70 SBD (PDB entry 4po2; Zhang et al., 2014) from which the substrate peptide had been removed as a molecularreplacement model. Iterative cycles of refinement and model building were performed using BUSTER (Bricogne et al., 2017) and Coot (Emsley et al., 2010), respectively (statistics are given in Table 4). Figures were prepared using PyMOL (DeLano, 2002). Analysis of complex interfaces was performed by PDBePISA (Krissinel \& Henrick, 2007). Sequence alignments were performed by Clustal Omega (Madeira et al., 2019).

\section{Results and discussion}

\subsection{Structure of the PfHsp70-x SBD-substrate complex}

The asymmetric unit of the PfHsp70-x SBD-substrate crystals comprised four copies of the complex, with each protomer adopting the characteristic $\operatorname{SBD} \beta / \operatorname{SBD} \alpha$ subdomain architecture of Hsp70 SBDs (Fig. 1a). PfHsp70-x $\operatorname{SBD} \beta$ (residues 424-539) is made up of eight $\beta$-strands arranged into two $\beta$-sheets of four antiparallel strands, and is connected by a short loop, $\mathrm{L}_{\alpha, \beta}$, to SBD $\alpha$ (residues 540-644). SBD $\alpha$ consists of five $\alpha$-helices, which first cape $\operatorname{SBD} \beta$ and are then arranged into a C-terminal antiparallel three-helical bundle. In this manner, $\operatorname{SBD} \alpha$ forms a helical lid across $\operatorname{SBD} \beta$, thereby potentially regulating access to the substrate-binding site 
(see below). The contact between the $\operatorname{SBD} \beta$ and $\operatorname{SBD} \alpha$ subdomains showed that our crystallographic structure corresponds to the closed state of this substrate-binding module. The four PfHsp70-x SBD monomers in the asymmetric unit were structurally similar, with an average pairwise $\mathrm{C}^{\alpha}$ root-mean-square deviation (r.m.s.d.) of $2.19 \AA$ (215 aligned atoms with no outlier rejections; Fig. 1a); however, the similarity was significantly more pronounced within the SBD subdomains ( $\mathrm{SBD} \beta, 0.76 \AA$ average pairwise $\mathrm{C}^{\alpha}$ r.m.s.d. for 105 aligned atoms, Fig. $1 b$; $\mathrm{SBD} \alpha, 1.12 \AA$ average pairwise $\mathrm{C}^{\alpha}$ r.m.s.d. for 104 aligned atoms, Fig. 1c). This suggested that the greatest plasticity of PfHsp70-x SBD manifests in the relative positioning of $\operatorname{SBD} \beta$ and $\operatorname{SBD} \alpha$, which is consistent with these two subdomains being mobile relative to each other as part of the chaperone catalytic cycle.

The PfHsp70-x SBD $\beta$ harboured the substrate-binding site primarily between loops $\mathrm{L}_{1,2}$ and $\mathrm{L}_{3,4}$ (Fig. $2 a$ ), which defined a hydrophobic groove. Residues Ala437 and Tyr462 of $\mathrm{L}_{1,2}$ and $\mathrm{L}_{3,4}$, respectively, formed an arch over this groove, thereby inhibiting substrate release (Fig. 2b). All four copies of PfHsp70-x SBD in the asymmetric unit were complexed with a substrate peptide with sequence $\mathrm{N}_{1}$ RLLLTG $_{7}$; in three of the complexes all seven peptide residues could be resolved (Fig. 2a), while only four residues $\left(\mathrm{Leu}_{4}-\mathrm{Gly}_{7}\right)$ could be built for the last copy of the complex. Substrate binding was stabilized by the burial of $\mathrm{Leu}_{5}$ of the peptide in a hydrophobic pocket formed by Leu434, Phe459, Val469, Ile471, Ile505 and Val507 of $\operatorname{SBD} \beta$ (Fig. 2c), as well as additional hydrophobic interactions between $\mathrm{Leu}_{4}$ and $\mathrm{Thr}_{6}$ of the the peptide and the Ala437-Tyr462 arch of $\operatorname{SBD} \beta$ (Fig. $2 b$ ). Although the low resolution of this structure limits the accuracy with which hydrogen bonds can be inferred, we note that up to eight such bonds may form between the peptide $\mathrm{Leu}_{3}-\mathrm{Gly}_{7}$ backbone atoms and the $\operatorname{SBD} \beta$ backbone (Ala437, Thr460, Tyr462 and Leu470) or side-chain (Gln466 and Gln 491) moieties (Fig. 2d). Complex formation buried $\sim 567 \AA^{2}$ of solvent-accessible surface area on average in the three PfHsp70-x SBD-substrate copies where all peptide residues could be resolved; thus, the complex interface accounts for nearly $50 \%$ of the total accessible peptide area. However, it should be noted that the NRLLLTG model peptide may not capture all of the interactions formed by the true substrates of the PfHsp70-x SBD.

\subsection{Comparison of PfHsp70-x SBD with other Hsp70s}

Comparison of SBDs from Hsp70 homologues revealed a high level of amino-acid sequence identity (Supplementary Fig. S1), in particular for $\operatorname{SBD} \beta$. Crucially, the residues of the hydrophobic substrate-binding interface were nearly identical in human erythrocytic Hsp70s (Hsp70 and Hsc70; Bryk \& Wiśniewski, 2017) and PfHsp70-x, and also in the more sequence-remote E. coli DnaK. However, Ala437 and Tyr462 of PfHsp70-x, which form the substrate $\operatorname{arch}$ in $\operatorname{SBD} \beta$, were identical in human chaperones, but their relative size was reversed (Met instead of Ala and Ala instead of Tyr) in DnaK. This reversal of amino-acid size in PfHsp70-x versus bacterial DnaK was previously recognized from the amino-acid sequence and may impact substrate specificity and release (Shonhai et al., 2007; Zhu et al., 1996). Further, the $\mathrm{L}_{\alpha, \beta}$ loop was identical in the erythrocytic and $P$. falciparum chaperones, while it differed in the equivalent segment of DnaK by having larger amino acids (-TNDK- compared with -ASSG-) and

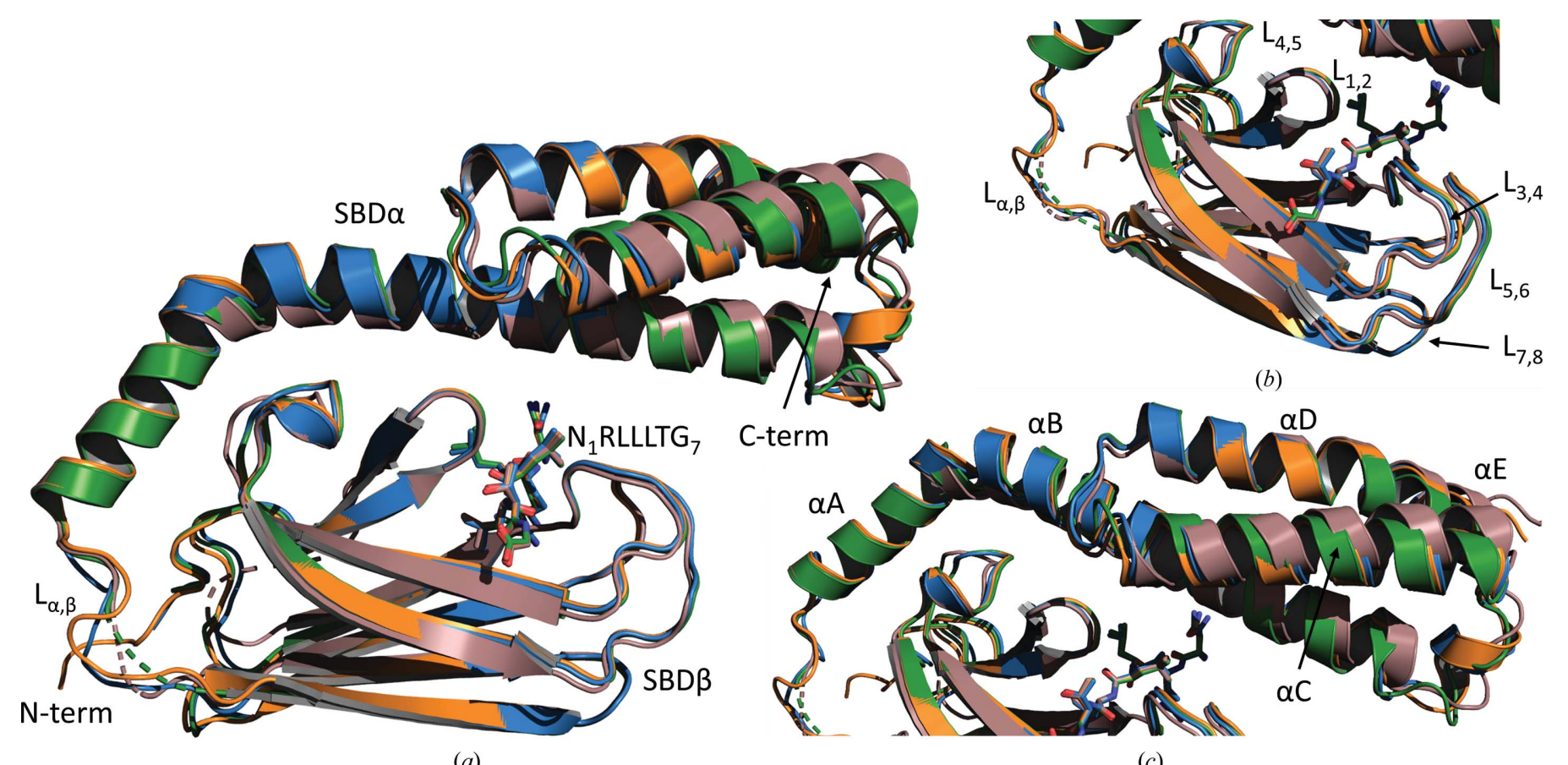

Figure 1

(a)

(c)

Structure of the PfHsp70-x SBD-substrate complex. (a) Superposition of the four complex copies in the asymmetric unit of the crystal, showing the protein architecture comprising $\mathrm{SBD} \beta$ and $\mathrm{SBD} \alpha$ subdomains. The substrate peptide $\left(\mathrm{N}_{1} \mathrm{RLLLTG}_{7}\right)$ is shown as sticks. $(b, c)$ Close-up views of the $\mathrm{SBD} \beta$ and $\operatorname{SBD} \alpha$ subdomains, respectively. $\operatorname{SBD} \beta \beta$-strand-connecting loops and $\operatorname{SBD} \alpha$ helices are enumerated. 

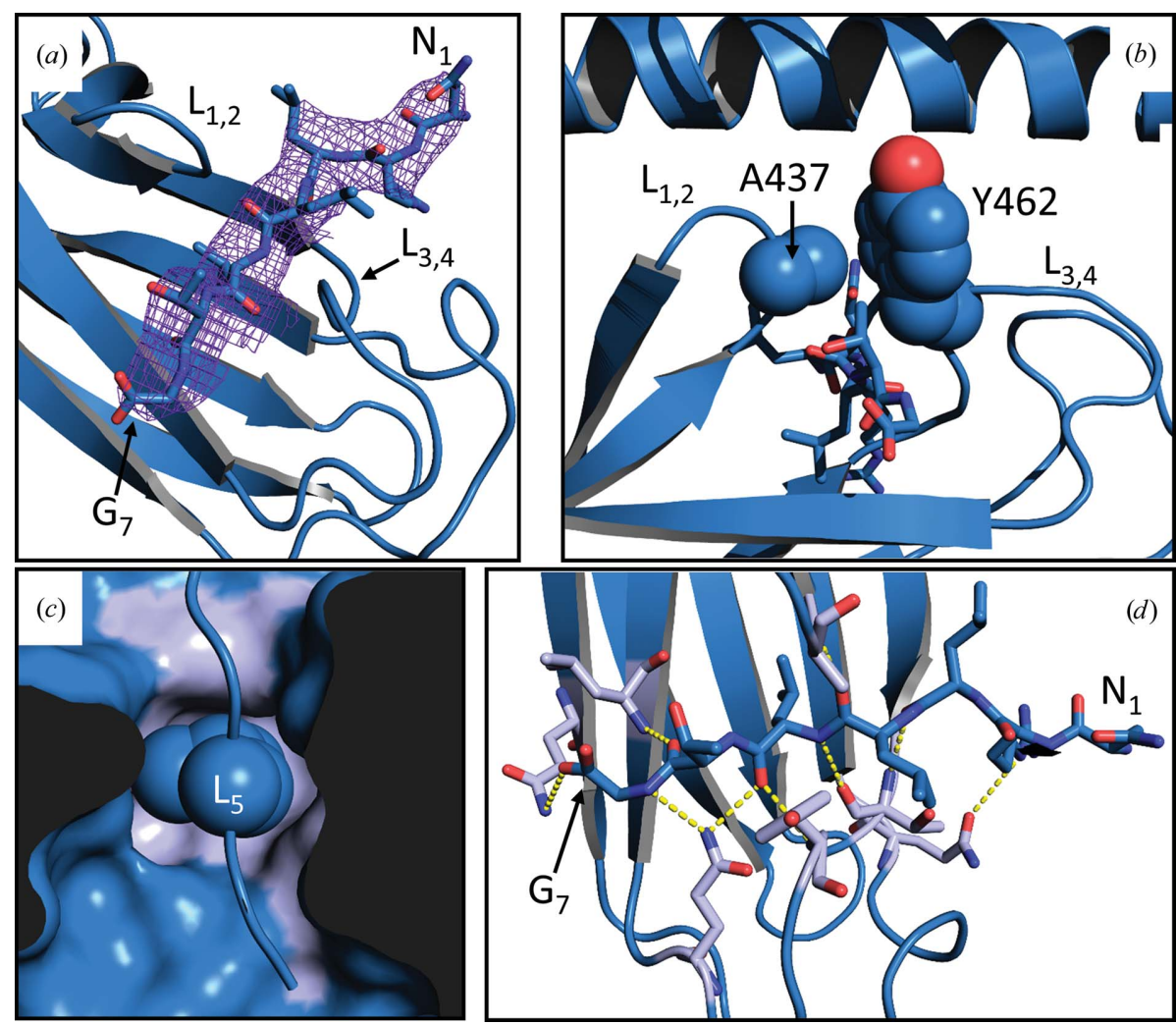

Figure 2

Substrate recognition by PfHsp70-x SBD. (a) Electron-density $\left(2 F_{\mathrm{o}}-F_{\mathrm{c}}\right)$ OMIT map of the substrate peptide bound to PfHsp70-x SBD. The map was contoured at $1 \sigma$. The protein loops $\mathrm{L}_{1,2}$ and $\mathrm{L}_{3,4}$, which define the substrate-binding groove, are indicated. The substrate peptide, which is shown as a reference but was not included in the map calculations, is represented as sticks. (b) The Ala437-Tyr462 arch forming over the substrate-binding groove is shown. The side chains of the protein residues forming this arch are denoted as spheres. (c) Surface representation of the PfHsp70-x SBD hydrophobic cavity where the peptide $\mathrm{L}_{5}$ (side chain shown as spheres) docks. The surfaces of the protein residues that are directly involved in $\mathrm{L}_{5}$ binding are coloured light blue. $(d)$ Hydrogen bonds inferred between the substrate peptide and PfHsp70-x SBD. Protein residues participating in hydrogen bonds are shown as sticks and coloured light blue. Hydrogen bonds are shown as dashed yellow lines.

being one residue longer. As opening and closing motions of the $\operatorname{SBD} \beta / \mathrm{SBD} \alpha$ subdomains along the $\mathrm{L}_{\alpha, \beta}$ loop have been shown to affect substrate binding and chaperone activity (Zhuravleva et al., 2012; Kityk et al., 2012), we concluded that PfHsp70-x SBD is likely to be functionally closer to its human erythrocytic counterparts than the prokaryotic chaperone.

The extent of the similarity of PfHsp70-x SBD to the human chaperones was underscored by structural comparisons. Superposition of the SBDs of human Hsp70 (PDB entry 4po2; Zhang et al., 2014), DnaK (PDB entry 1dkz; Zhu et al., 1996) and PfHsp70-x revealed broadly similar three-dimensional structures (Fig. 3). Nevertheless, alignment of the PfHsp70-x SBD with that of human Hsp70 showed a $\mathrm{C}^{\alpha}$ r.m.s.d. of $2.45 \AA$ on average (219 atoms aligned), whereas comparison of PfHsp70-x SBD with DnaK revealed a $\mathrm{C}^{\alpha}$ r.m.s.d. of $3.03 \AA$ (212 atoms aligned). Thus, the SBD of PfHsp70-x is structurally more similar to that of human Hsp70 than to E. coli DnaK. However, it is worth noting that the complexed state of Hsp70 SBD domains is generally structurally conserved, with an average $\mathrm{C}^{\alpha}$ r.m.s.d. of only $\sim 1.3 \AA$ seen in comparisons of DnaK, E. coli HscA (PDB entry 1u00; Cupp-Vickery et al., 2004), human Hsp70 and bovine Hsc70 (PDB entry 3c7n; Schuermann et al., 2008).

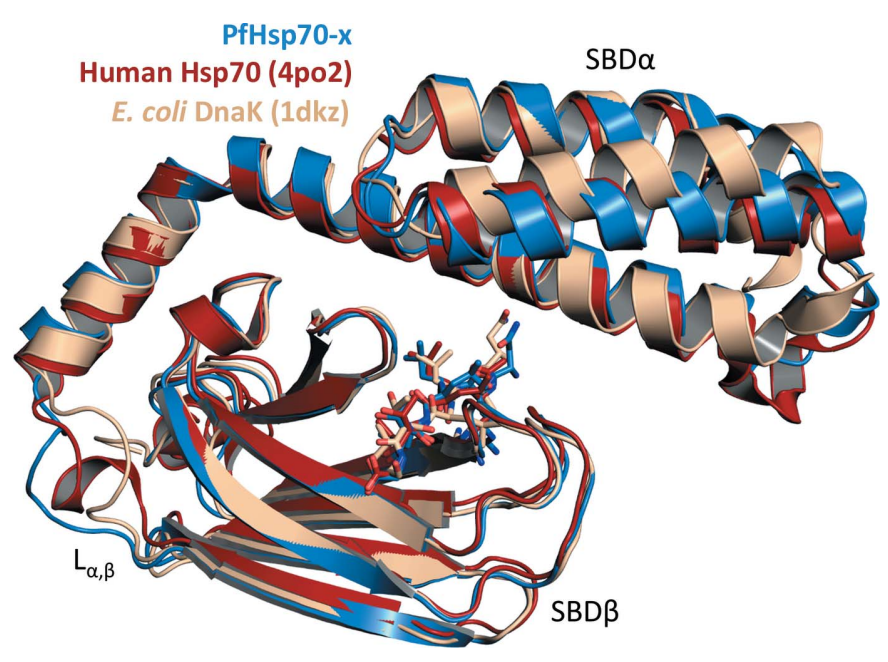

Figure 3

Comparison of PfHsp70-x SBD with homologues. Shown here is a structural superposition of PfHsp70-x SBD (blue) with the equivalent domains of human Hsp70 (red; PDB entry 4po2; Zhang et al., 2014) and E. coli DnaK (wheat; PDB entry 1dkz; Zhu et al., 1996). PfHsp70-x SBD is substantially similar to both of these domains; however, it is structurally closer to the human chaperone than to the prokaryotic counterpart. 
In conclusion, here we report the first crystallographic structure of the substrate-binding domain of PfHsp70-x. Analysis of the structure, and of the way in which a model substrate peptide was bound, suggested that PfHsp70-x adopts a canonical Hsp70-class chaperone SBD architecture. However, the PfHsp70-x SBD was more similar to those of human chaperones, such as those found in the erythrocyte, than to a prokaryotic counterpart. Although substrate selectivity depends on cross-talk between the Hsp70 SBD and ATP-binding domains (Swain et al., 2007; Vogel et al., 2006), which cannot be evaluated by the SBD structure alone, on the basis of similarities between the human Hsp70 and parasite Hsp70-x SBDs we propose that the parasite may be able to use human chaperones to supplement PfHsp70-x function, for example in maintaining the structure of parasite components exported to the host cell or for trafficking PfEMP1. Such a supplementary role of human Hsp70s may explain why the deletion or depletion of PfHsp70-x has only a partial impact on parasite survival and virulence (Charnaud et al., 2017; Day et al., 2019).

\section{Acknowledgements}

We thank Anastassia Kantsadi and Nada Mohamad for helpful discussions, Jemma Day for the PfHsp70-x SBD genetic construct and Edward Lowe for maintenance of the Oxford Biochemistry crystallization facility. We thank Diamond Light Source for the provision of synchrotronradiation facilities.

\section{Funding information}

Funding for this research was provided by: Medical Research Council (grant No. MR/N009274/1 to Ioannis Vakonakis); European Commission, Erasmus+ (studentship to Julia Schmidt).

\section{References}

Boorstein, W. R., Ziegelhoffer, T. \& Craig, E. A. (1994). J. Mol. Evol. 38, 1-17.

Bricogne, G., Blanc, E., Brandl, M., Flensburg, C., Keller, P., Paciorek, W., Roversi, P., Sharff, A., Smart, O. S., Vonrhein, C. \& Womack, T. O. (2017). BUSTER. Global Phasing Ltd, Cambridge, UK.

Bryk, A. H. \& Wiśniewski, J. R. (2017). J. Proteome Res. 16, $2752-$ 2761.

Charnaud, S. C., Dixon, M. W. A., Nie, C. Q., Chappell, L., Sanders, P. R., Nebl, T., Hanssen, E., Berriman, M., Chan, J. A., Blanch, A. J., Beeson, J. G., Rayner, J. C., Przyborski, J. M., Tilley, L., Crabb, B. S. \& Gilson, P. R. (2017). PLoS One, 12, e0181656.

Craig, A. G., Khairul, M. F. \& Patil, P. R. (2012). Malays. J. Med. Sci. 19, 5-18.

Cupp-Vickery, J. R., Peterson, J. C., Ta, D. T. \& Vickery, L. E. (2004). J. Mol. Biol. 342, 1265-1278.

Daniyan, M. O., Boshoff, A., Prinsloo, E., Pesce, E. R. \& Blatch, G. L. (2016). PLoS One, 11, e0148517.
Day, J., Passecker, A., Beck, H. P. \& Vakonakis, I. (2019). FASEB J. 33, 14611-14624.

DeLano, W. L. (2002). PyMOL. http://www.pymol.org.

Emsley, P., Lohkamp, B., Scott, W. G. \& Cowtan, K. (2010). Acta Cryst. D66, 486-501.

Goldberg, D. E. \& Cowman, A. F. (2010). Nat. Rev. Microbiol. 8, 617621.

Hviid, L. \& Jensen, A. T. (2015). Adv. Parasitol. 88, 51-84.

Kityk, R., Kopp, J., Sinning, I. \& Mayer, M. P. (2012). Mol. Cell, 48 , 863-874.

Koning-Ward, T. F. de, Dixon, M. W., Tilley, L. \& Gilson, P. R. (2016). Nat. Rev. Microbiol. 14, 494-507.

Krissinel, E. \& Henrick, K. (2007). J. Mol. Biol. 372, 774-797.

Külzer, S., Charnaud, S., Dagan, T., Riedel, J., Mandal, P., Pesce, E. R., Blatch, G. L., Crabb, B. S., Gilson, P. R. \& Przyborski, J. M. (2012). Cell. Microbiol. 14, 1784-1795.

Madeira, F., Park, Y. M., Lee, J., Buso, N., Gur, T., Madhusoodanan, N., Basutkar, P., Tivey, A. R. N., Potter, S. C., Finn, R. D. \& Lopez, R. (2019). Nucleic Acids Res. 47, W636-W641.

McCoy, A. J., Grosse-Kunstleve, R. W., Adams, P. D., Winn, M. D., Storoni, L. C. \& Read, R. J. (2007). J. Appl. Cryst. 40, 658-674.

Meimaridou, E., Gooljar, S. B. \& Chapple, J. P. (2009). J. Mol. Endocrinol. 42, 1-9.

Pace, C. N., Vajdos, F., Fee, L., Grimsley, G. \& Gray, T. (1995). Protein Sci. 4, 2411-2423.

Proellocks, N. I., Coppel, R. L., Mohandas, N. \& Cooke, B. M. (2016). Adv. Parasitol. 91, 1-86.

Rogala, K. B., Dynes, N. J., Hatzopoulos, G. N., Yan, J., Pong, S. K., Robinson, C. V., Deane, C. M., Gönczy, P. \& Vakonakis, I. (2015). eLife, 4, e07410.

Schuermann, J. P., Jiang, J., Cuellar, J., Llorca, O., Wang, L., Gimenez, L. E., Jin, S., Taylor, A. B., Demeler, B., Morano, K. A., Hart, P. J., Valpuesta, J. M., Lafer, E. M. \& Sousa, R. (2008). Mol. Cell, 31, 232-243.

Shonhai, A., Boshoff, A. \& Blatch, G. L. (2007). Protein Sci. 16, $1803-$ 1818.

Smith, J. D., Rowe, J. A., Higgins, M. K. \& Lavstsen, T. (2013). Cell. Microbiol. 15, 1976-1983.

Spillman, N. J., Beck, J. R. \& Goldberg, D. E. (2015). Annu. Rev. Biochem. 84, 813-841.

Swain, J. F., Dinler, G., Sivendran, R., Montgomery, D. L., Stotz, M. \& Gierasch, L. M. (2007). Mol. Cell, 26, 27-39.

Vogel, M., Mayer, M. P. \& Bukau, B. (2006). J. Biol. Chem. 281, 38705-38711.

Warncke, J. D., Vakonakis, I. \& Beck, H.-P. (2016). Microbiol. Mol. Biol. Rev. 80, 905-927.

Winter, G. (2010). J. Appl. Cryst. 43, 186-190.

Winter, G., Waterman, D. G., Parkhurst, J. M., Brewster, A. S., Gildea, R. J., Gerstel, M., Fuentes-Montero, L., Vollmar, M., MichelsClark, T., Young, I. D., Sauter, N. K. \& Evans, G. (2018). Acta Cryst. D74, 85-97.

World Health Organization (2019). World Malaria Report 2019. https://www.who.int/malaria/publications/world-malaria-report-2019/ en/.

Zhang, P., Leu, J. I.-J., Murphy, M. E., George, D. L. \& Marmorstein, R. (2014). PLoS One, 9, e103518.

Zhang, Q., Ma, C., Oberli, A., Zinz, A., Engels, S. \& Przyborski, J. M. (2017). Sci. Rep. 7, 42188.

Zhu, X., Zhao, X., Burkholder, W. F., Gragerov, A., Ogata, C. M., Gottesman, M. E. \& Hendrickson, W. A. (1996). Science, 272, 16061614.

Zhuravleva, A., Clerico, E. M. \& Gierasch, L. M. (2012). Cell, 151, 1296-1307. 